\title{
Correction to: Database Systems for Advanced Applications
}

Jian Pei, Yannis Manolopoulos, Shazia Sadiq, and Jianxin Li

\section{Correction to: \\ J. Pei et al. (Eds.): Database Systems for Advanced Applications, LNCS 10827, https://doi.org/10.1007/978-3-319-91452-7}

The original version of this chapter titled "Free-Rider Episode Screening via Dual Partition Model" contained the following three mistakes:

1. In table 1, row 2, column 3, the average occurrence per event on STK dataset was "1.037". It should be " 1,037 ".

2. The last model name in the legend of Figure 3 was "EIP". It should be "EDP".

3. In the experiment part the stock symbols and their companies were confused.

In the updated version these mistakes were corrected.

In the originally published version of chapters titled "BASSI: Balance and Status Combined Signed Network Embedding" and "Sample Location Selection for Efficient Distance-Aware Influence Maximization in Geo-Social Networks" the funding information in the acknowledgement section was incomplete. This has now been corrected.

The updated version of these chapters can be found at https://doi.org/10.1007/978-3-319-91452-7_4

https://doi.org/10.1007/978-3-319-91452-7_24

https://doi.org/10.1007/978-3-319-91452-7_43 